\title{
Method to reconstruct neuronal action potential train from two-photon calcium imaging
}

\author{
Tingwei Quan \\ Huazhong University of Science and Technology \\ Britton Chance Center for Biomedical Photonics \\ Wuhan National Laboratory for Optoelectronics \\ Wuhan 430074, China \\ and \\ Hubei University of Education \\ College of Mathematics and Economics \\ Wuhan 430205, China
}

\section{Xiuli Liu \\ Xiaohua Lv \\ Huazhong University of Science and Technology Britton Chance Center for Biomedical Photonics Wuhan National Laboratory for Optoelectronics Wuhan 430074, China}

\section{Wei R. Chen}

University of Texas Health Science Center at Houston Department of Neurobiology and Anatomy Houston, Texas 77030

\author{
Shaoqun Zeng \\ Huazhong University of Science and Technology \\ Britton Chance Center for Biomedical Photonics \\ Wuhan National Laboratory for Optoelectronics \\ Wuhan 430074, China
}

\begin{abstract}
Identification of a small population of neuronal action potentials (APs) firing is considered essential to discover the operating principles of neuronal circuits. A promising method is to indirectly monitor the AP discharges in neurons from the recordings their intracellular calcium fluorescence transients. However, it is hard to reveal the nonlinear relationship between neuronal calcium fluorescence transients and the corresponding AP burst discharging. We propose a method to reconstruct the neuronal AP train from calcium fluorescence diversifications based on a multiscale filter and a convolution operation. Results of experimental data processing show that the false-positive rate and the event detection rate are about 10 and $90 \%$, respectively. Meanwhile, the APs firing at a high frequency up to $40 \mathrm{~Hz}$ can also be successfully identified. From the results, it can be concluded that the method has strong power to reconstruct a neuronal AP train from a burst firing. (C) 2010 Society of Photo-Optical Instrumentation Engineers. [DOI: 10.1117/1.3505021]
\end{abstract}

Keywords: action potential firing; multiscale filter; calcium fluorescence transient; template convolution.

Paper 10275R received May 26, 2010; revised manuscript received Aug. 12, 2010; accepted for publication Sep. 10, 2010; published online Nov. 5, 2010.

\section{Introduction}

Spatiotemporal dynamic patterns of local neural circuits are essential for discovering the mechanisms of the nervous system. ${ }^{1-4}$ Recently, with the advances in calcium indictors and the twophoton microscopy technique, reconstructions of the action potential (AP) discharge in local neural circuits from their simultaneously recorded calcium fluorescence transients in vivo and in vitro were carried out. ${ }^{5-9}$ Furthermore, two-photon fluorescence random scanning microscopy has been designed and constructed, through which calcium fluorescence transients sampled at about a $1-\mathrm{kHz}$ frequency from neuronal local circuits can be recorded synchronously. ${ }^{10-12}$ These data at a high sampling rate enable us to reconstruct AP firing of local neuronal circuits at a millisecond-scale precision. ${ }^{13}$ Although the neuronal burst discharge carries more coding information than a single AP firing, ${ }^{14-16}$ revealing neuronal AP burst trains from the corresponding calcium waves is well-known as hard work. ${ }^{17-20}$ This is so not only because burst firing is caused by the large amount of depolarization ${ }^{15,16}$ and has not been clearly defined, but also because neuronal calcium fluorescence transients always have a nonlinear relationship with their APs firing, causing the diversities and complexities of calcium fluorescence waves. ${ }^{21}$

Address all correspondence to: Xiuli Liu, Wuhan National Laboratory for Optoelectronics, Huazhong University of Science and Technology, Wuhan 430074, China. Tel: 86-27-87792033-303; Fax: 86-27-87792034; E-mail: xlliu@mail.hust.edu.cn.
Practically, ${ }^{14-16}$ some burst firing sequences consist of APs with intervals of less than $20 \mathrm{~ms}$.

Driven by the necessity to reveal the principles of neuronal circuits, many valuable methods to reconstruct AP sequences from their corresponding calcium fluorescence transients have been designed. For example, the template-matching method was developed to detect a single AP discharge from a calcium fluorescence transient, which works well whenever neurons discharge sparsely. ${ }^{22,23}$ The uncorrected ${ }^{24}$ and corrected first-derivative method including deconvolution ${ }^{7,25,26}$ can provide reliable neuronal firing rates, but are limited to inferring neuronal AP trains from calcium fluorescence transients with a low signal-to-noise ratio (SNR), defined as the ratio of the amplitude of the template to the standard deviation of noise in the initial calcium waves. Some statistical methods such as clustering ${ }^{27}$ and a combination of principal component analysis and a support vector machine, ${ }^{28}$ can only partially describe the calcium wave diversities that derive from the neuronal irregularly discharging at a high frequency rate. Some sophisticated methods $s^{9,29,30}$ are suitable for reconstructing the AP train from a burst firing. However, compared to the methods just mentioned, more setting parameters or hypotheses may confine their applications. Recently, a method termed "peeling" was proposed for this purpose, but a fixed template matching the rising duration is still necessary. ${ }^{13}$

1083-3668/2010/15(6)/066002/6/\$25.00 (C) 2010 SPIE 
For the calcium fluorescence transients at a high frequency $(1 \mathrm{kHz})$, the nonlinear calcium dynamics triggered by neuronal burst firing have become increasingly obvious and are difficult to describe using a single model or several patterns. In addition, the SNR of fluorescence signals is a decisive factor for the accuracy of the reconstruction. Therefore, aimed to reconstruct the AP burst firing trains from calcium fluorescence transient with relative low SNRs, a method based on multiscale filter combined with template convolution (MSF_TC) is proposed. First, the parts of calcium fluorescence transients corresponding to neuronal AP firing are identified using a multiscale filter, and the AP train candidates are generated. Then, we combine the AP train candidates with the template function, depending on the fitting degrees with the original calcium waves, to confirm AP firing timing sequences.

To illustrate the MSF_TC algorithm performance, this method was applied in processing real data sets from our optical system. ${ }^{11,12}$ The reconstruction results of the proposed method and that of two typical methods, the template-matching method and the deconvolution method, were compared. The reconstruction results were further analyzed to emphasize some important features of the MSF_TC algorithm.

\section{Methods}

\subsection{Recording Calcium Fluorescence Transients}

\subsubsection{Sample preparing and fluorescence labeling}

Slices were made from postnatal (P)14-P20 Wistar rats. The animals were anesthetized with $1 \%$ sodium phentobarbital $(50 \mathrm{mg} / \mathrm{kg})$. After fast decapitation, the brain was dissected rapidly and placed in ice-cold oxygenated $(95 \%$ O2 and 5\% CO2) ACSF (artificial cerebrospinal fluid) containing (in $\mathrm{mM}$ ) $119 \mathrm{NaCl}, 2.5 \mathrm{KCl}, 2.5 \mathrm{CaCl} 2,1.3 \mathrm{MgCl}$, $1 \mathrm{NaH} 2 \mathrm{PO} 4,26 \mathrm{NaHCO} 3$, and 11 glucose. Cortical and hippocampal slices, 350- to $400-\mu \mathrm{m}$ thick, were cut with a vibratome (VTS1000, Leica) and maintained in an incubation chamber for at least $1 \mathrm{~h}$ at room temperature (22 to $25^{\circ} \mathrm{C}$ ) before recording. During the experiments, the slice was transferred to a submersion-recording chamber and incubated in oxygenated ACSF (1 to $3.0 \mathrm{ml} / \mathrm{min}$ ) at room temperature.

After $1 \mathrm{~h}$ in the incubation chamber, the slices were transferred to a recording chamber, where they were stained with calcium indicators. As in whole-cell patch recording, all local population cells were performed sequentially by the glass micropipette and maintained about 20 min to result in reliable and rapid loading of somata and subsequent complete labeling of dendritic and axonal arborizations. Bath-applied 4-AP at a final concentration of $100 \mu \mathrm{M}$ was used to induce epileptiform discharging.

\subsubsection{Imaging system for calcium fluorescence transients}

A custom-constructed random access two-photon fluorescence microscope system was used to image the calcium fluorescence transients. The setup of the system has been described in detail by Lv et al. ${ }^{11}$ Briefly, a Ti:sapphire laser (Tsunami, Spectra Physics) was pumped by a solid state $\mathrm{cw}$ laser (Millennia
Xs, Spectra Physics) to produce a mode-locked laser output ${ }^{31}$ ( $800 \mathrm{~nm}, \sim 100$-fs pulse width at $80 \mathrm{MHz}$ repetitive frequency). Laser intensity was adjusted with an electro-optic modulator (EOM). After the dispersion compensation prism (tilted at 45 deg), two orthogonal acousto-optic deflectors (AODs) were used for $x-y$ deflection of the laser beam. Then, the laser beam was coupled to an upright microscope (BX61WI, Olympus). A water immersion objective $40 \times$ (Olympus) with a working distance of $3.3 \mathrm{~mm}$, and a numerical aperture 0.80 was used for imaging. The imaging process was controlled by software custom-written in LabVIEW.

\subsubsection{Simultaneous recording of calcium fluorescence transients and AP train}

To compare the real with the reconstructed AP train of a single neuron, neuronal calcium fluorescence transients and AP firing were recorded simultaneously. Calcium fluorescence transients was expressed as $\Delta F / F=\left(F-F_{0}\right) / F_{0}$, where $F_{0}$ is the resting calcium fluorescence signal, and $F$ are the calcium fluorescence intensities. In this paper, the dwelling time of each region of interest (ROI) is $250 \mu \mathrm{s}$, and four ROIs were preselected, so the random access sampling rate is $1 \mathrm{kHz}$. Whole-cell patch recording was obtained to monitor membrane voltage of neurons using a patch clamp amplifier (EPC-9, HEKA). Recording pipettes were routinely filled with a solution containing (in $\mathrm{mM}$ ) $125 \mathrm{~K}$-gluconate, $15 \mathrm{KCl}, 10$ Hepes, $4 \mathrm{MgCl}_{2}, 3 \mathrm{Mg}$-ATP, $0.3 \mathrm{Na}-\mathrm{GTP}, 10 \mathrm{Na}_{2}$-phosphocreatine, 0.2 EGTA; and $100 \mu \mathrm{M}$ Fluo-5F (pH 7.3 with $\mathrm{KOH}, 290$ to $300 \mathrm{mOsm}$ ). Signals were sampled at $10 \mathrm{kHz}$ by the amplifier system.

\subsection{Reconstructing Neuronal AP Train}

The MSF_TC algorithm contains three steps. The first step is the extraction of the template, which is then used in the following two steps. The AP train candidates are generated in the second step. Last, through template convolution, the optimal AP train is confirmed and regarded as the reconstruction results.

\subsubsection{Extraction of the template}

The template extraction is primarily based on the fact that the calcium wave triggered by single AP remains almost unchanged. $^{32,33}$ Thus, 10 to 20 calcium waves from individual calcium fluorescence transients are aligned at the time when they are ascending most rapidly, and then averaged. The averaged trace is then regarded as the template. For demonstration in Figs. 1(d) to 1(g) a template is used to be convoluted with the AP train. Note that the value of the template baseline should be rectified to zero.

\subsubsection{AP train candidates generation}

In this step, identification of a valid calcium fluorescence transient and the extraction of its rising duration are necessary. The 10- and 100-Hz low-pass Butterworth filtered calcium fluorescence transients sampled at $1 \mathrm{kHz}$ were used to identify a valid signal and reconstruct the AP train, respectively, as shown in Figs. 1(a) and 1(b). The number of zeros and poles in the Butterworth filter were set to be 3 and 10, respectively, at all times. The rising and decay duration triggered by a single AP firing usually last hundreds of milliseconds. Using the $10-\mathrm{Hz}$ low-pass filter, the AP firing and corresponding rising durations 
(a)

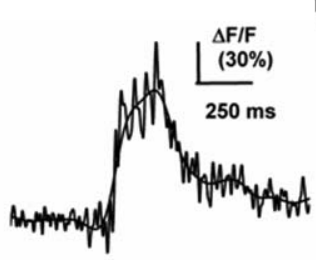

(d)
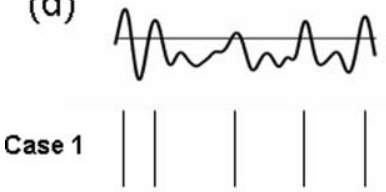

(f)

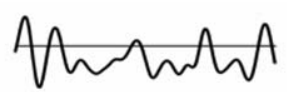

Case 2 (b)

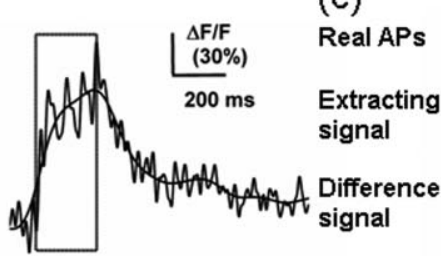

(e)
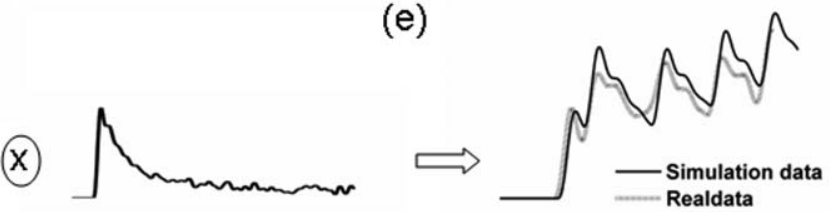

(g)

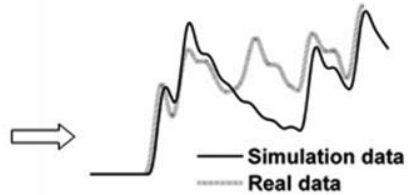

Fig. 1 MSF_TC algorithm for AP train reconstruction. (a) calcium fluorescence transients after 10- and 100-Hz low-pass filtering, respectively. (b) Identify the valid calcium fluorescence transient and extract its rising duration [the box in (b)]. (c) The extracted rising duration (middle) corresponds to 5 APs (top), and the predicted APs firing at the peak of the differential of the rising duration of the calcium waves, as indicated by the arrows (bottom). Note that the peak should be more than the given threshold (horizontal line in the bottom panel). Five predicted APs can generate 32 AP train candidates according to Eq. (4). (d) and (f) Two of 32 AP train candidates that convolved with the template and simulated signals were generated. The AP train is regarded as a reconstructed AP train, which corresponds to the simulated signal that has the most similar real signal. Based on this, the AP train in (d) is the reconstructed AP train rather than that in (f).

are easily identified. The identified arising duration, the adjacent decay duration, and the following 500-ms duration consist of a part of the calcium fluorescence transients [Fig. 1(b)], the time interval of which corresponds to the $100-\mathrm{Hz}$ filtered signal. The corresponding signal is used to reconstruct the AP train, which is mostly based on the consideration that the rising duration trigged by two APs firing at a $25-\mathrm{ms}$ interval can still be completely separated [Fig. 1(c)] using a 100-Hz low-pass filter. Accordingly, MSF_TC can successfully identify APs firing at frequencies up to $40 \mathrm{~Hz}$ from the calcium fluorescence transients.

As indicated in the box in Fig. 1(b) and the middle panel of Fig. 1(c), the extracted rising duration $p(t) t \in\left[t_{i}, t_{i+1+2 k}\right]$ from the identified valid signal could meet the following criteria:

$$
p\left(t_{i+1}\right)-p\left(t_{i}\right)>\text { thr, }
$$

$p\left(t_{i+1+2 m}\right)<\max \left[p\left(t_{i+3+2 m}\right), p\left(t_{i+5+2 m}\right), p\left(t_{i+7+2 m}\right)\right]$

$$
m=0,1, \ldots, k-1,
$$

$p\left(t_{i+1+2 k}\right)>\max \left[p\left(t_{i+1+2 k}\right), p\left(t_{i+5+2 k}\right), p\left(t_{i+7+2 k}\right)\right]$,

where $t_{i}$ is the time corresponding to the peak or valley value $p\left(t_{i}\right)$, and thr denotes the threshold determined by clustering for the amplitudes of the noise and the calcium waves triggered by a single AP. A suitable setting is 0.6 to 0.7 times the amplitude of the template. The times $\left[t_{1}, t_{2}, \ldots, t_{k}\right]$, indicated by the arrows in the bottom panel of Fig. 1(c), correspond to the peaks of the differential of the extracted rising duration [Fig. 1(c), middle], and the peak values are larger than the standard deviation of the differential signal [horizontal line in Fig. 1(c), bottom panel]. These times are used to generate AP train candidates, as follows:

$$
\ell_{s}(t)= \begin{cases}1 \text { or } 2 & t=t_{1} \\ 0 \text { or } 1 & t=t_{i}(i>1) \\ 0 & \text { otherwise }\end{cases}
$$

where $\ell_{s}(t)$ is a candidate of AP train, $s=1,2, \ldots, 2^{k}$. Here, $\ell_{s}\left(t_{i}\right)=1$ means that single AP fires at time $t_{i}$. Note that $\ell_{s}\left(t_{1}\right)=2$ represents a special AP burst firing pattern that two AP fire at an about 10-ms time interval and their waves are not completely separated. This usually happens at the beginning of the burst. ${ }^{34,35}$

\subsubsection{Template convolution}

The AP candidate train $\ell_{s}(t)$ was convolved with the template to get the corresponding simulating signal $f_{s}(t)$. As shown in Figs. 1(d) and 1(e), the simulated signal [solid line in the Fig. 1(e)] was obtained by convoluting the AP train candidate containing five APs [Fig. 1(d)] with the template. The difference between the simulated signal $f_{s}(t)$ and the extracted rising duration $p(t)$ $t \in\left[t_{i}, t_{i+1+2 k}\right]$ is measured by the Euclidean distance shown in

$$
k_{s}=\min _{i}\left\{\operatorname{norm}\left[p_{[t, t+\Delta t]}, f_{s}(i, i+\Delta t)\right]\right\} .
$$

The minimum value $k_{s}{ }^{*}$ indicates that the simulating signal $f_{s}{ }^{*}(t)$ is the most similar to the rising duration. Thus, the $\mathrm{AP}$ train $\ell_{s}^{*}(t)$ corresponding to $f_{s}^{*}(t)$ is regarded as reconstructed AP train. As illustrated in Figs. 1(d) through $1(\mathrm{~g})$, the AP train candidate in Fig. 1(d) is more suitable to represent the real AP train than that in Fig. 1(f). 


\section{Results}

The MSF_TC algorithm was applied to eight pieces of experimental data from four different cells. They are $300 \mathrm{~s}$ long and sampled at a $1-\mathrm{kHz}$ frequency. The template of any trace was extracted from the individual data sets. The difference between the reconstructed and real AP trains reflecting the efficiency of the MSF_TC algorithm was weighed with false positive rates and event detection rates.

In Fig. 2(a), the data set 1 (top) contains 301 APs, and the visual comparison of real (middle) and reconstruction (bottom) AP train indicated that no difference between them exists. In this case, the false positive rate and the event detection rate were 6 to 11 and 94 to $95 \%$, respectively, when the threshold was set to be 0.6 to 0.7 times the amplitude of the template, as indicted in Figs. 2(e) and 2(f) (labeled the number 1 on the $x$ axis). As shown in Figs. 2(e) and 2(f), the reconstruction results from the other seven pieces of data were similar with that already considered.

We also measured the time precision of the AP train reconstruction by comparing it with the real AP train. The absolute error of AP firing estimated timing and real timing distributed over the 0 - to $10-\mathrm{ms}$ interval was more than $90 \%$ [Fig. 2(b)]. The standard deviation of the absolute errors was less than $7 \mathrm{~ms}$ for data set 1 , and was also suitable for the other seven data sets [Fig. 2(g)].

To show the ability of the MSF_TC algorithm to reconstruct the burst firing, a histogram of the inter-AP interval (ISI) less than 1000 ms was given by analyzing data set 1 [Fig. 2(c)]. From the histogram, we can conclude that high-frequency and short-duration firing are common patterns in our data set. For further verification, ISI distribution from eight AP trains was also given and ISIs less than $50 \mathrm{~ms}$ held $30 \%$ [Fig. 2(h)]. In addition, a part of calcium fluorescence transient triggered by a burst firing is shown in Fig. 2(d). During the AP burst firing, the rising duration generated by individual AP obviously changes, which heavily hinders the reconstruction of AP train using some characteristics of a calcium fluorescence transient, such as its rising amplitude and the amplitude variance. Considered from the reconstruction results and our data set features, the MSF_TC algorithm can effectively identify an individual AP from a burst.

To test the robustness of the MSF_TC algorithm against the threshold, a $10 \%$ change in the threshold was applied, namely, the threshold increasing from 0.6 to 0.7 times of the amplitude of the template. The false positive rate (FPR) and the event detection rate (EVR) were increased and decreased by an average of about 3\%, respectively [Figs. 2(e) and 2(f)].

We compared the reconstruction performance of the MSF_TC algorithm with that of two other typical methods, template matching 22,23 and the deconvolution $\operatorname{method}^{7}$ (Table 1). The calculations, including parameter selection, were carried out in accordance with previous work. ${ }^{7,22,23}$ For MSF_TC, the threshold value was set to 0.7 times the amplitude of the template. Low EDRs demonstrate that the template matching is unsuitable for reconstructing the neuronal burst firing. For the deconvolution method, the effective results are strongly dependent on the high SNR. For example, the SNRs of data sets 1 to 4 are about a range of from 2 to 2.5 ; in contrast, the data set 6 reaches 4 . Therefore, compared with the deconvolution method, the MSF_TC algorithm could provide effective results from the calcium transients even with a relatively low SNR.

\section{Discussion and Conclusions}

As already noted, the neuronal bursts firings are extremely important for information encoding. We proposed a simple method to reconstruct an AP train from the calcium fluorescence
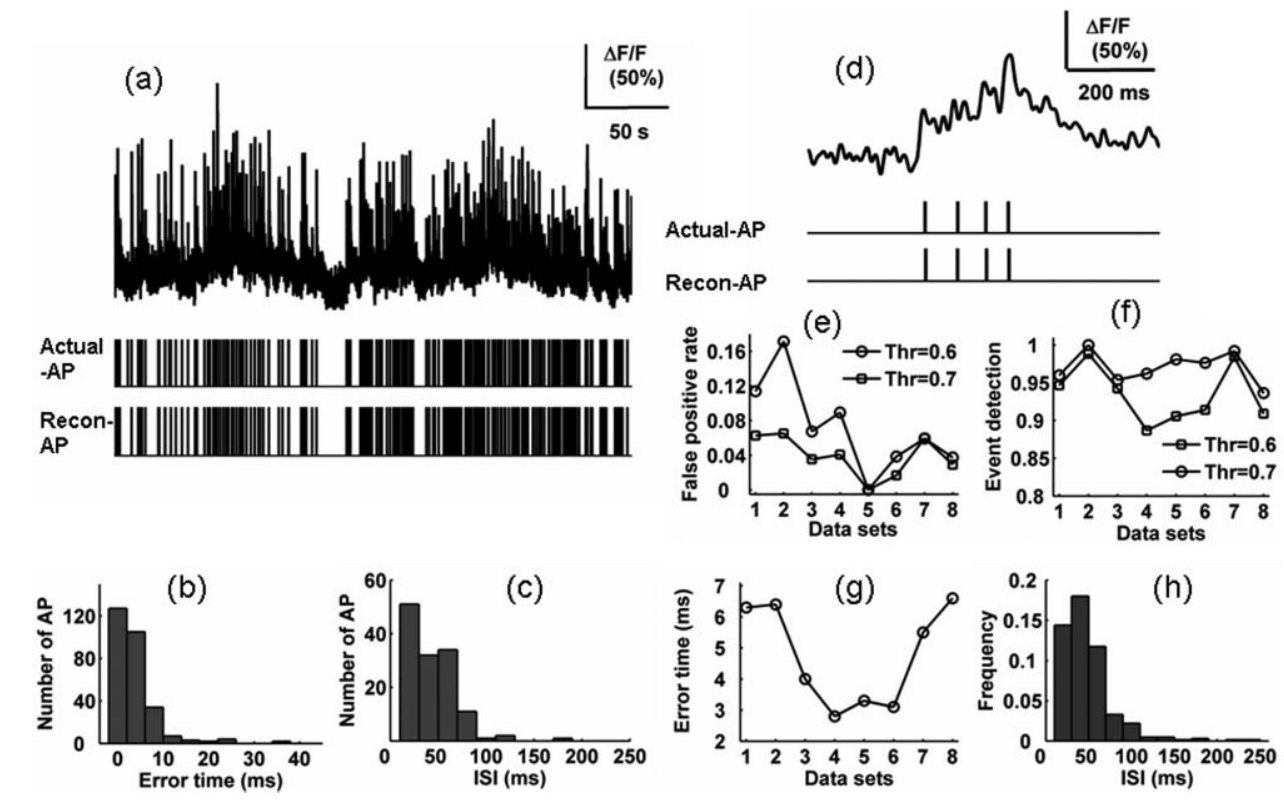

Fig. 2 Capability of MSF_TC algorithm in AP train reconstruction: (a) calcium fluorescence transients (top), the corresponding real (Middle) and reconstructed (bottom) AP trains; (b) histogram of the difference between AP firing reconstruction and real timing obtained by analyzing data set in (a); (c) ISI distribution from the actual AP train [middle panel in (a)]; and (d) burst AP firing corresponding calcium fluorescence, real and reconstructed AP train. For an eight-data-set analysis, a 10\% change of the threshold influenced by (e) the false positive rate and ( $\mathrm{f}$ ) the event detection. (g) plot of the standard deviation of the absolute error between real and reconstructed AP timings and (h) ISI distribution for eight real AP trains. 
Table 1 MSF_TC algorithm compared with template matching and the deconvolution method.

\begin{tabular}{|c|c|c|c|c|c|c|}
\hline \multirow{2}{*}{$\begin{array}{l}\text { Data } \\
\text { Set }\end{array}$} & \multicolumn{2}{|c|}{$\begin{array}{l}\text { MSF_TC } \\
\text { Algorithm }\end{array}$} & \multicolumn{2}{|c|}{ Template Matching } & \multicolumn{2}{|c|}{ Deconvolution } \\
\hline & FPR $(\%)$ & $\operatorname{EDR}(\%)$ & FPR $(\%)$ & EDR (\%) & FPR $(\%)$ & EDR $(\%$ \\
\hline 1 & 6 & 95 & 18 & 38 & 2 & 65 \\
\hline 2 & 7 & 99 & 28 & 70 & 6 & 67 \\
\hline 3 & 4 & 94 & 30 & 70 & 7 & 70 \\
\hline 4 & 4 & 89 & 37 & 75 & 8 & 83 \\
\hline 5 & 0 & 91 & 25 & 74 & 17 & 100 \\
\hline 6 & 2 & 92 & 19 & 33 & 5 & 98 \\
\hline 7 & 6 & 98 & 12 & 44 & 3 & 93 \\
\hline 8 & 3 & 91 & 18 & 43 & 20 & 100 \\
\hline
\end{tabular}

transients that is suitable for even neuronal burst firing. By the multiscale filter application and valid calcium identification, the influence of long-duration noise on the reconstruction result is eliminated to a large extent. Furthermore, the simulation signals generated by the convolution of AP train candidates with the template can provide many patterns to match neuronal burst firing. These procedures guarantee the reliability and effectiveness of the MSF_TC algorithm in the analysis of the data sets.

Note that the method requires a proper template, so acquiring the template is an important procedure. Calcium fluorescence transients induced by a single AP is an all or nothing process. ${ }^{36}$ In addition, the model for computing calcium dynamics demonstrates that the amplitude of the calcium fluorescence transient is almost constant. ${ }^{21}$ Therefore, the template can be successfully obtained by extracting and averaging the calcium traces generated by a single AP.

In the MSF_TC algorithm, multiscale filters are adopted. The scale selection is determined by the sum of the rise and decay duration formed by single AP firing and the noise type. There is a significant difference between the AP firing and no AP firing, corresponding to a $10-\mathrm{Hz}$ low-pass-filtered calcium fluorescence transient. Thus, identifying valid calcium fluorescence is easy and the threshold is not discussed. In addition, properly increasing the length of the valid calcium fluorescence transient prevents missing the APs, whose firings lead to calcium wave part separations. Obviously, identifying an individual AP from a burst is impossible using the $10-\mathrm{Hz}$ filtered signal. To resolve the rising durations triggered by two APs with 25-ms firing intervals, that is, the two rising durations are completely separate$100-\mathrm{Hz}$ low-pass filters were properly selected, as verified by our results. Note that, in part of our data sets, the occurrence of two APs with an interval of $20 \mathrm{~ms}$ can cause the complete separation of the rising duration. As a result, the APs firing at a $50-\mathrm{Hz}$ frequency from a burst were successfully identified.

Compared with other typical methods, i.e., template matching ${ }^{22}$ and the deconvolution method, ${ }^{7}$ the results illustrate that MSF_TC algorithm is superior to these two meth- ods. The reason may be as follows: many of the burst firings corresponding to the calcium fluorescence transients lead the template matching to have a low efficacy and an outstanding performance of the deconvolution method is strongly dependent on a high SNR. For sparse AP firing, the MSF_TC algorithm is still inferior to template matching, because it chooses only the characteristics of the rising duration that identify an individual AP from a burst firing. Furthermore, like the other methods, 9, 29,30 the MSF_TC algorithm can not provide the AP firing rate when the rising durations are not separated. Properly use of decay duration may enhance the efficiency of identifying individual APs from a burst firing.

In summary, according to the characteristics of the data sets from our optical system, a reliable method was designed to reconstruct AP trains from calcium fluorescence transients. The results showed that the MSF_TC algorithm has a perfect performance in revealing an individual AP from calcium fluorescence transients corresponding to a burst firing, which should be beneficial for further deciphering neuronal circuits with a higher temporal resolution.

\section{Acknowledgments}

This work was supported by the National Natural Science Foundation of China (Grant Nos. 30927001 and 30925013). WRC was supported by National Institutes of Health (NIH) Grants DC009666 and DC003918.

\section{Reference}

1. C. Stosiek, O. Garaschuk, K. Holthoff, and A. Konnerth, "In vivo two-photon calcium imaging of neuronal networks," Proc. Natl. Acad. Sci. U.S.A. 100(12), 7319-7324 (2003).

2. G. J. Augustine, F. Santamaria, and K. Tanaka, "Local calcium signaling in neurons," Neuron 40(2), 331-346 (2003).

3. K. Ohki, S. Chung, Y. H. Ch'Ng, P. Kara, and R. C. Reid, "Functional imaging with cellular resolution reveals precise micro-architecture in visual cortex," Nature 433(7026), 597-603 (2005).

4. N. L. Rochefort, H. Jia, and A. Konnerth, "Calcium imaging in the living brain: prospects for molecular medicine," Trends Mol. Med. 14(9), 389-399 (2008).

5. W. Denk, J. H. Strickler, and W. W. Webb, "Two-photon laser scanning fluorescence microscopy," Science 248(4951), 73-76 (1990).

6. X. Liu, H. Gong, X. Li, and W. Zhou, "Monitoring calcium concentration in neurons with cameleon," J. Biosci. Bioeng. 105(2), 106-109 (2008).

7. E. Yaksi and R. W. Friedrich, "Reconstruction of firing rate changes across neuronal populations by temporally deconvolved $\mathrm{Ca} 2+$ imaging," Nature Meth. 3(5), 377-383 (2006).

8. D. A. Dombeck, A. N. Khabbaz, F. Collman, T. L. Adelman, and D. W. Tank, "Imaging large-scale neural activity with cellular resolution in awake, mobile mice,"Neuron 56(1), 43-57 (2007).

9. D. S. Greenberg, A. R. Houweling, and J. Kerr, "Population imaging of ongoing neuronal activity in the visual cortex of awake rats," Nature Neurosci. 11(7), 749-751 (2008).

10. R. Salome, Y. Kremer, S. Dieudonne, J. F. Leger, O. Krichevsky, C. Wyart, D. Chatenay, and L. Bourdieu, "Ultrafast random-access scanning in two-photon microscopy using acousto-optic deflectors," J. Neurosci. Meth. 154(1-2), 161-174 (2006).

11. X. Lv, C. Zhan, S. Zeng, W. R. Chen, and Q. Luo, "Construction of multiphoton laser scanning microscope based on dual-axis acousto-optic deflector," Rev. Sci. Instrum. 77(4), 046101 (2006).

12. S. Zeng, X. Lv, K. Bi, C. Zhan, D. Li, W. R. Chen, W. Xiong, S. L. Jacques, and Q. Luo, "Analysis of the dispersion compensation of acousto-optic deflectors used for multiphoton imaging," J. Biomed. Opt. 12, 24015 (2007). 
13. B. F. Grewe, D. Langer, H. Kasper, B. M. Kampa and F. Helmchen, "High-speed in vivo calcium imaging reveals neuronal network activity with near-millisecond precision," Nature Meth. 7(5), 399-405 (2010).

14. A. A. Grace and B. S. Bunney, "The control of firing pattern in nigral dopamine neurons: burst firing," J. Neurosci. 4(11), 2877-2890 (1984).

15. J. E. Lisman, "Bursts as a unit of neural information: making unreliable synapses reliable," Trends Neurosci. 20(1), 38-43 (1997).

16. K. D. Harris, H. Hirase, X. Leinekugel, D. A. Henze, and G. Buzsáki, "Temporal interaction between single spikes and complex spike bursts in hippocampal pyramidal cells," Neuron 32(1), 141-149 (2001).

17. V. Iyer, T. M. Hoogland, and P. Saggau, "Fast functional imaging of single neurons using random-access multiphoton (RAMP) microscopy," J. Neurophysiol. 95(1), 535-545 (2006).

18. Y. Wu, X. Liu, W. Zhou, X. Lv, and S. Zeng, "Observing neuronal activities with random access two-photon microscope," J. Innov. Opt. Health Sci. 12(1), 67-71 (2009).

19. W. G. Bel and F. Helmchen, "In vivo calcium imaging of neural network function," Physiology (Bethesda) 22, 358-365 (2007).

20. B. F. Grewe and F. Helmchen, "Optical probing of neuronal ensemble activity," Curr. Opin. Neurobiol. 19(5), 520-529 (2009).

21. E. Neher and G. J. Augustine, "Calcium gradients and buffers in bovine chromaffin cells," J. Physiol. 450(1), 273-301 (1992).

22. J. D. Clements and J. M. Bekkers, "Detection of spontaneous synaptic events with an optimally scaled template," Biophys. J. 73(1), 220-229 (1997).

23. J. Kerr, D. Greenberg, and F. Helmchen, "Imaging input and output of neocortical networks in vivo," Proc. Natl. Acad. Sci. U.S.A. 102(39), 14063-14068 (2005).

24. D. Smetters, A. Majewska, and R. Yuste, "Detecting action potentials in neuronal populations with calcium imaging," Methods 18(2), 215-221 (1999).

25. L. Moreaux and G. Laurent, "Estimating firing rates from calcium signals in locust projection neurons in vivo," Front. Neural Circ. 1, 2 (2007).
26. E. A. Mukamel, A. Nimmerjahn, and M. J. Schnitzer, "Automated analysis of cellular signals from large-scale calcium imaging data," Neuron 63(6), 747-760 (2009).

27. T. Sato, N. Gray, Z. Mainen, and K. Svoboda, "The functional microarchitecture of the mouse barrel cortex," Plos Biol. 5(7), 1440-1452 (2007).

28. T. Sasaki, N. Takahashi, N. Matsuki, and Y. Ikegaya, "Fast and accurate detection of action potentials from somatic calcium fluctuations," J. Neurophysiol. 100(3), 1668-1676 (2008).

29. J. T. Vogelstein, B. O. Watson, A. M. Packer, R. Yuste, B. Jedynak, and L. Paninski, "Spike inference from calcium imaging using sequential monte carlo methods," Biophys. J. 97(2), 636-655 (2009).

30. Y. Mishchencko, J. T. Vogelstein, and L. Paninski, "A Bayesian approach for inferring neuronal connectivity from calcium fluorescence imaging data," Ann. Appl. Stat. (in press).

31. W. Watanabe, S. Matsunaga, K. Fukui, and K. Itoh, "Intracellular manipulation by femtosecond lasers: review," J. Innov. Opt. Health Sci. 2(1), 1-8 (2009).

32. M. Marhl, S. Schuster, and M. Brumen, "Mitochondria as an important factor in the maintenance of constant amplitudes of cytosolic calcium oscillations," Biophys. Chem. 71(2-3), 125-132 (1998).

33. D. J. Wallace, S. M. Zum Alten Borgloh, S. Astori, Y. Yang, M. Bausen, S. Kügler, A. E. Palmer, R. Y. Tsien, R. Sprengel, and J. Kerr, "Single-spike detection in vitro and in vivo with a genetic $\mathrm{Ca}^{2+}$ sensor," Nature Meth. 5(9), 797-804 (2008).

34. P. Perreault and M. Avoli, "4-Aminopyridine-induced epileptiform activity and a GABA-mediated long-lasting depolarization in the rat hippocampus," J. Neurosci. 12(1), 104-115 (1992).

35. P. Salazar, R. Tapia, and M. A. Rogawski, "Effects of neurosteroids on epileptiform activity induced by picrotoxin and 4-aminopyridine in the rat hippocampal slice," Epilepsy Res. 55(1-2), 71-82 (2003).

36. Y. M. Usachev and S. A. Thayer, "All-or-none $\mathrm{Ca}^{2+}$ release from intracellular stores triggered by $\mathrm{Ca}^{2+}$ influx through voltage-gated $\mathrm{Ca}^{2+}$ channels in rat sensory neurons," J. Neurosci. 17(19), 7404-7414 (1997). 